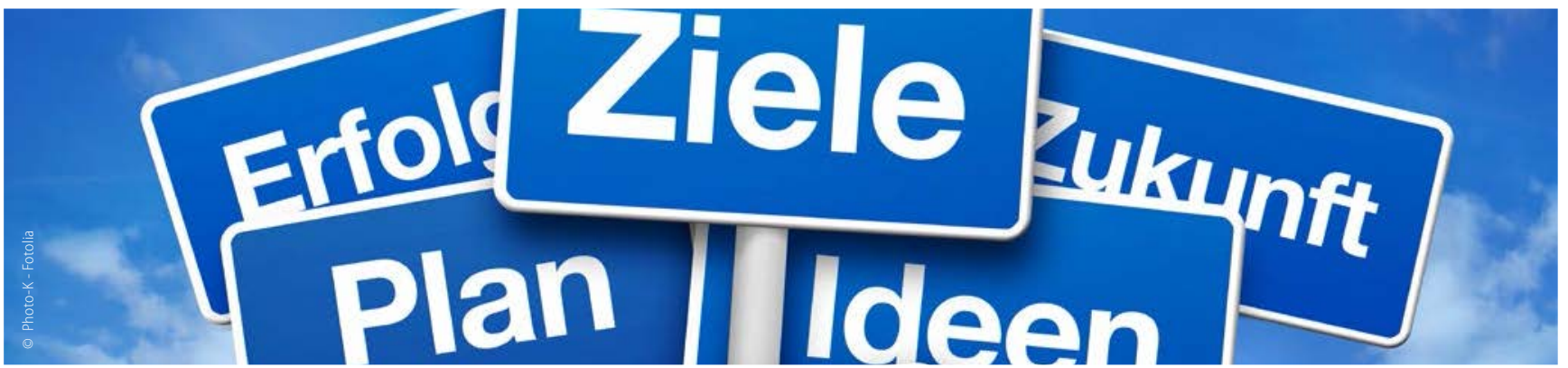

Neuausrichtung und Modernisierung

\title{
Projekt „Zukunftssicherung“
}

Liebe Kolleginnen und Kollegen,

vor zwei Jahren stimmte eine große Mehrheit im Freien Verband Deutscher Zahnärzte für den Richtungswechsel. Der Verband brauchte und braucht eine Neuausrichtung, um sich aus festgefahrenen Strukturen zu befreien, verbandsinterne Streitigkeiten beizulegen und sich umfassend zu modernisieren. Ich stehe für diesen Richtungswechsel - dafür bin ich gewählt worden. Das ist der Weg, den der Bundesvorstand und ich gemeinsam begonnen haben und den wir erfolgreich weiterführen wollen.

Die Aufgabe, vor der wir als überzeugte Freiberufler standen und stehen, ist klar umrissen: Der Verband muss zukunftssicher aufgestellt werden. Dem gesellschaftlichen Wandel und den neuen Ansprüchen der Generation junger Zahnärzte, müssen wir Rechnung tragen. Dazu gehört es, Ideen zuzulassen, die über die klassische freiberufliche Einzelpraxis hinausgehen, moderne Berufsausübungsmodelle zu entwickeln und zu unterstützen und dabei unseren Grundwerten der Freiberuflichkeit treu zu bleiben. Wir haben begonnen, die Veränderungen aufzunehmen und neue Perspektiven für unsere Mitglieder zu schaffen.

\section{Herausforderung der Gesundheitspolitik}

$\mathrm{Zu}$ unserem Projekt „Zukunftssicherung" gehört, dass wir uns mit den sich ändernden politischen Umständen intensiv auseinandersetzen. Zahlreiche Gesetze der Großen Koalition zeigen deutlich in Richtung Verstaatlichung und Überregulierung des Gesundheitswesens. Wir haben aktiv Stellung bezogen und kämpfen gegen diese politischen Strangulierungsversuche unserer Freiberuflichkeit. Und nicht nur in Deutschland greift der Staat die Freiberuflichkeit an, auch Europa gewinnt immer mehr Einfluss auf unseren Berufsstand. Es sind die Richtlinien oder Verordnungen, die primär nicht auf das Gesundheitssystem zielen, die uns Sorgen bereiten. Über die Binnenmarktregelungen greift Europa immer stärker auf unsere Praxen zu. Dem Primat des freien Austauschs von Personen, Waren und Dienstleistungen folgt Schritt für Schritt die Deregulierung des Berufsstandes. In einer globalisierten Welt, in der unsere Werte kein Konsens mehr sind, gilt es umso mehr, das zu schützen, wofür wir stehen.

Dafür muss der Berufsstand auf Augenhöhe mit der Politik reden, um nicht abgehängt zu werden. Mit pauschalen Ablehnungen ohne Lösungsangebote finden wir weder in Berlin noch in Brüssel Gehör. Nur der sachkundige und inhaltliche Dialog führt zu einer gleichberechtigten Koexistenz auf der politischen Bühne. Diesen kritischen Dialog haben wir aufgenommen, mit der Politik, mit der BZÄK und der KZBV. Ich bin der tiefen Über- zeugung, dass sich der FVDZ nach außen öffnen muss, um bestehen zu können - und um gehört zu werden.

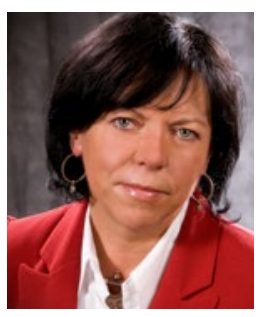

\section{Innere Veränderung durch Modernisierung}

Dazu gehört es auch, Strukturen kritisch zu hinterfragen, zu durchbrechen und Neues zu wagen. Wir haben unsere innere Struktur verändert, um nach außen schlagkräftiger reagieren zu können. Als moderner Verband, ob für den Berufseinsteiger oder den niedergelassenen Zahnarzt, können wir nur dann attraktiv sein, wenn wir nach innen wie nach außen das Feuer der Begeisterung für die Freiberuflichkeit wecken und erhalten. Die Begeisterung für die Freiheit des eigenverantwortlichen Handelns. Das ist eine unserer großen Aufgaben. Für unsere Mitglieder und für jene, die es werden wollen, bietet der Verband Leistungen an, die überzeugen. Unser Erfolgsrezept: Wir hören zu, was Absolventen und junge Zahnärzte uns zu sagen haben und antworten mit der Erfahrung von 60 Jahren Freiberuflichkeit. Wir hören aber ebenso zu, wenn es um Probleme und Fragen der erfahrenen Kollegen geht. Daraus ist ein großes Generationenprojekt entstanden, das in den Mittelpunkt stellt, wofür wir alle stehen: die freiberufliche Praxis in eigener Verantwortung. Um den FVDZ zukunftsfähig zu machen, ist das eine der Säulen im Fundament des Verbandes.

Für unsere politischen Ziele und für unsere Modernisierung im Inneren braucht es einen starken FVDZ. Wir haben in den vergangenen zwei Jahren gehandelt und vieles auf den Weg gebracht. Das Projekt „Zukunftssicherung“ läuft.

Ihre

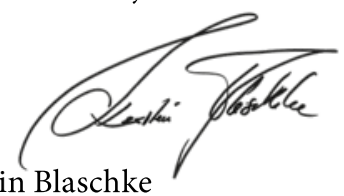

Kerstin Blaschke

\section{Der Bundesvorstand im Interview}

Seit knapp zwei Jahren leitet Kerstin Blaschke als Bundesvorsitzende den Freien Verband Deutscher Zahnärzte - und hat dem FVDZ eine Art Frischzellenkur verordnet: neue Köpfe, neue Themen, neuer Stil. Einen Monat vor der FVDZ-Hauptversammlung zieht Blaschke eine Zwischenbilanz: „Ein gutes Stück Arbeit ist geschafft, aber es war bisher nur der Anfang." Die DFZRedakteurinnen Melanie Fügner und Sabine Schmitt haben in diesem Heft die Mitglieder des Bundesvorstandes zu ihren Arbeitsbereichen befragt. 